\title{
An analytical formula for VIX futures and its applications
}

\author{
Song-Ping Zhu, Guang-Hua Lian
}

\author{
School of Mathematics and Applied Statistics \\ University of Wollongong, Australia
}

\begin{abstract}
In this paper we present a closed-form, exact solution for the pricing of VIX futures in a stochastic volatility model with simultaneous jumps in both the asset price and volatility processes. The newly-derived formula is then used to show that the well-known convexity correction approximations can sometimes lead to large errors. Utilizing the newly-derived formula, we also conduct an empirical study, the results of which demonstrate that the Heston stochastic volatility model is a good candidate for the pricing of VIX futures. While incorporating jumps into the underlying price can further improve the pricing of VIX futures, adding jumps to the volatility process appears to contribute little improvement for pricing VIX futures.
\end{abstract}

Key Words: VIX futures, Heston model, jump diffusions, characteristic function

Acknowledgment: The authors gratefully acknowledge the proof-read of the entire manuscript by, as well as many valuable comments from, Prof. Robert J. Elliott of the University of Adelaide, Australia.

The manuscript was received in Aug. 2009, and accepted in Dec. 2010.

\footnotetext{
*Correspondence author, Professor Song-Ping Zhu, School of Mathematics and Applied Statistics, University of Wollongong, Wollongong, N.S.W. 2522, Australia. Phone: 61-2-42213807, fax: 61-2-42214845, e-mail: spz@uow.edu.au.
} 


\section{Introduction}

The Volatility Index (VIX) has been considered as the world's benchmark for stock market volatility since its introduction in 1993 by the CBOE (Chicago Board Options Exchange). In 2003, CBOE switched into a new methodology to define the VIX. Shortly after the revision of the VIX definition, on March 26, 2004, the CBOE Futures Exchange started to trade the firstever futures contracts on the VIX Index, and in February 2006 VIX options were launched. Binary options on VIX also began trading in 2008. Very recently, mini-VIX futures were launched in 2009. The rapid development of VIX derivative products manifests their economic importance.

With the growing popularity of VIX derivatives, considerable research has been drawn to the development of appropriate pricing models for VIX derivatives. Grunbichler \& Longstaff (1996) first developed a pricing model for volatility futures and volatility options based on a mean-reverting squared-root volatility process. Carr \& Wu (2006) presented a lower bound and an upper bound for the price of VIX futures. Zhang \& Zhu (2006) proposed an expression for VIX futures, assuming the S\&P500 is described by Heston (1993)'s stochastic volatility model. Zhu \& Zhang (2007) further derived a no-arbitrage pricing model for VIX futures based on the variance term structure. Lin (2007) presented a convexity adjustment approximation for the value of the VIX futures under various stochastic volatility models with simultaneous jumps, both in the asset price and variance processes. Psychoyios et al. (2007) provided a pricing model for both VIX futures and VIX options based on a squared root mean reverting process with jumps. Sepp $(2008 a, 2008 b)$ applied the square root stochastic variance model with variance jumps to describe the evolution of S\&P500 volatility, and demonstrated how to apply the model to the pricing and hedging of VIX futures and options.

More recently, Lin \& Chang (2009) derived a pricing formula for VIX options under the stochastic volatility model with simultaneous jumps both in the S\&P500 and its related variance processes, and empirically examined the performance of each generalization of the S\&P500 dynamics in improving VIX option pricing. Zhang et al. (2010) used market data to establish the relationship between the VIX and the VIX futures prices, and then established a theoretical relationship between VIX futures and VIX, using a simple square root mean-reverting process with a stochastic long-term mean. Zhang \& Huang (2010) analyzed the market data of the CBOE 
S\&P500 three-month variance futures, and established a linear relation between the price of fixed time-to-maturity variance futures and the VIX ${ }^{2}$ by using a simple mean-reverting stochastic volatility model for the S\&P500 index. Lu \& Zhu (2009) derived a new pricing framework for VIX futures, and then used a Kalman filter and maximum likelihood method for model parameter estimations and comparisons. They provided evidence that a third factor is statistically significant for variance term structure dynamics. Luo \& Zhang (2010) proposed a general twofactor stochastic volatility framework for VIX, and gave an empirical analysis showing that the framework is good at both capturing time-series dynamics of VIX and generating a rich crosssectional shape for the term structure. Some other typical recent papers about the VIX and its derivatives (futures and options) include Wang \& Daigler (2010), Lin \& Chang (2010), Cont \& Kokholm (2010). Carr \& Lee (2009) provided a comprehensive overview of the research in this area, suggesting that studies on the valuation of VIX derivatives are far from conclusive.

This study aims to accomplish three main tasks. Firstly, a closed-form and exact pricing formula is developed to evaluate the VIX futures in a general framework that allows for stochastic volatility, random jumps in the underlying asset, and random jumps in the variance process. Our solution procedure is demonstrated through a proposition in the first part of Section 2. Secondly, utilizing the newly-found formula, we then examine the accuracy of the well-known convexity correction approximations in pricing VIX futures (see for example, Lin 2007; Zhang et al. 2010) in the second part of Section 2. Through numerical comparison, we show that there are nontrivial differences between our results and those from Lin's (2007) approximation solution, and the third order approximation proposed in Zhang et al. (2010) performs even worse than Lin's (2007) approximate formula. Thirdly, we present an empirical study in Section 3, examining the effects of adding jumps to the underlying asset and its volatility processes. Using the joint time series data of S\&P500 and VIX, we demonstrate the determination of model parameters using the MCMC approach and show that the Heston stochastic volatility model captures the dynamics of the S\&P500 and is a good candidate for the pricing of VIX futures; adding jumps into the volatility process appears to give little improvement for pricing VIX futures. Finally, a brief summary is provided in Section 4. 


\section{VIX Futures Models}

The purpose of this section is to derive a closed-form formula for VIX futures, in the framework of stochastic volatility with jump-diffusion characteristics observed in the time-series literature. A closed-form exact solution in such a general framework enables us to closely scrutinize the accuracy of some approximate formulae in the literature, and to empirically examine the effect of adding jumps into models for pricing VIX futures.

\subsection{The Volatility Index}

The current VIX is calculated in a model-free manner as a weighted sum of out-of-money option prices across all available strikes on the S\&P500 index. As described in the CBOE white paper ${ }^{1}$, the new VIX, which is the underlying asset of VIX futures and options, is defined by means of $\mathrm{VIX}_{t}^{2}$

$$
\mathrm{VIX}_{t}^{2}=\left(\frac{2}{\bar{\tau}} \sum_{i} \frac{\Delta K_{i}}{K_{i}^{2}} e^{r \bar{\tau}} Q\left(K_{i}\right)-\frac{1}{\bar{\tau}}\left[\frac{F}{K_{0}}-1\right]^{2}\right) \times 100^{2},
$$

where $\bar{\tau}=\frac{30}{365}, K_{i}$ is the strike price of the i-th out-of-the-money option in the calculation, $F$ is the time-t forward index level, $Q\left(K_{i}\right)$ denotes the time-t midquote price of the out-of-the-money option at strike $K_{i}, K_{0}$ is the first strike below the forward index level, and $r$ denotes the time-t risk-free rate with maturity $\bar{\tau}$.

For a better understanding of the financial interpretation, this expression of the VIX squared can be given in terms of the risk-neutral expectation of the log contract, using a mathematical simplification (see Lin 2007; Duan \& Yeh 2010 for more details):

$$
\mathrm{VIX}_{t}^{2}=-\frac{2}{\bar{\tau}} E^{\mathbb{Q}}\left[\ln \left(\frac{S_{t+\bar{\tau}}}{F}\right) \mid \mathscr{F}_{t}\right] \times 100^{2}
$$

Here $\mathbb{Q}$ is the risk-neutral probability measure, $F=S_{t} e^{r \bar{\tau}}$ denotes the 30-day forward price of the underlying S\&P500 with a risk-free interest rate $r$ under the risk-neutral probability, and $\mathscr{F}_{t}$ is the filtration up to time $t$.

\footnotetext{
${ }^{1}$ see the white paper of VIX, available at http://www.cboe.com/micro/vix/vixwhite.pdf
} 


\subsection{Pricing VIX Futures}

Due to the relationship between $\mathrm{VIX}_{t}^{2}$ and S\&P500, as described in Equation (2), a natural

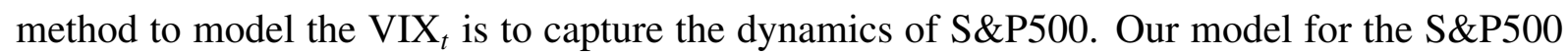
in this paper incorporates stochastic volatility and simultaneous jumps in both the asset price and the volatility process. This general model was initially proposed by Duffie et al. (2000). Under the physical probability measure $\mathbb{P}$, the $\mathrm{S} \& \mathrm{P} 500$ index, denoted by $S_{t}$, is assumed to have dynamics:

$$
\left\{\begin{array}{l}
d S_{t}=S_{t}\left(r_{t}+\gamma_{t}\right) d t+S_{t} \sqrt{V_{t}} d W_{t}^{S}+d\left(\sum_{n=1}^{N_{t}} S_{\tau_{n-}}\left[e^{Z_{n}^{S}}-1\right]\right)-S_{t} \bar{\mu} \lambda d t \\
d V_{t}=\kappa\left(\theta-V_{t}\right) d t+\sigma_{V} \sqrt{V_{t}} d W_{t}^{V}+d\left(\sum_{n=1}^{N_{t}} Z_{n}^{V}\right)
\end{array}\right.
$$

where $r_{t}$ is the constant spot interest rate, $V$ is the diffusion component of the variance of the underlying asset dynamics (conditional on no jumps occurring), $d W_{t}^{S}$ and $d W_{t}^{V}$ are two standard Brownian motions correlated with $E\left[d W_{t}^{S}, d W_{t}^{V}\right]=\rho d t, \kappa, \theta$ and $\sigma_{V}$ are, respectively, the meanreverting speed parameter, long-term mean, and variance coefficient of the diffusion $V_{t}$, and $N_{t}$ is an independent Poisson process with intensity $\lambda$. Possible jumps are taken into consideration in both the underlying dynamics $S_{t}$ and the variance process $V_{t}$, with the jump sizes being assumed to have a distribution $Z_{n}^{V} \sim \exp \left(\mu_{V}\right)$, and $Z_{n}^{S} \mid Z_{n}^{V} \sim N\left(\mu_{S}+\rho_{J} Z_{n}^{V}, \sigma_{S}^{2}\right) \cdot \bar{\mu}=e^{\mu_{S}+\frac{1}{2} \sigma_{S}^{2}} /\left(1-\rho_{J} \mu_{V}\right)-1$ is the risk premium of the jump term in the process to compensate the jump component, and $\gamma_{t}$ is the total equity premium.

Following the standard analysis in literature (e.g., Duffie et al. 2000; Pan 2002; Eraker 2004; Broadie et al. 2007), we represent the dynamics of the S\&P500 index, under the risk-neutral probability measure $\mathbb{Q}$, as:

$$
\left\{\begin{array}{l}
d S_{t}=S_{t} r_{t} d t+S_{t} \sqrt{V_{t}} d W_{t}^{S}(\mathbb{Q})+d\left(\sum_{n=1}^{N_{t}(\mathbb{Q})} S_{\tau_{n-}}\left[e^{Z_{n}^{S}(\mathbb{Q})}-1\right]\right)-S_{t} \bar{\mu}^{\mathbb{Q}} \lambda d t \\
d V_{t}=\kappa^{\mathbb{Q}}\left(\theta^{\mathbb{Q}}-V_{t}\right) d t+\sigma_{V} \sqrt{V_{t}} d W_{t}^{V}(\mathbb{Q})+d\left(\sum_{n=1}^{N_{t}(\mathbb{Q})} Z_{n}^{V}(\mathbb{Q})\right)
\end{array}\right.
$$

where $\bar{\mu}^{\mathbb{Q}}=e^{\mu_{S}^{\mathbb{Q}}+\frac{1}{2} \sigma_{S}^{2}} /\left(1-\rho_{J} \mu_{V}\right)-1$ and $\mu_{S}^{\mathbb{Q}}$ is the corresponding risk-neutral parameter of $\mu_{S}$. Consistent with the specification considered in Pan (2002) or Eraker (2004), the risk premium parameters in our study are: the diffusive volatility risk premium $\eta_{V}=\kappa^{\mathbb{Q}}-\kappa$ and the jump risk 
premium $\eta_{J}=\mu_{S}^{\mathbb{Q}}-\mu_{S}$. Notice that the $\sigma_{V}, \rho, \kappa \theta, \lambda$ and other jump parameters are the same under both the physical probability measure $\mathbb{P}$ and the risk-neutral probability measure $\mathbb{Q}$. The specification for diffusive volatility risk premium $\eta_{V}$ is standard in the literature, whereas there are various ways of specifying the measure changes (jump risk premium) for the jump processes. Broadie et al. (2007) considered a more general specification for the measure changes for the jump processes by allowing the jump intensity and all the jump parameters to change for the measures $\mathbb{P}$ and $\mathbb{Q}$.

As shown in Equation (2), VIX squared is just the conditional risk-neutral expectation of the log contract of the S\&P500 over the next 30 calendar days. Under the general specification of Equation (4), this expectation can be carried out explicitly, yielding

$$
\operatorname{VIX}_{t}^{2}=\left(a V_{t}+b\right) \times 100^{2}
$$

where

$$
\left\{\begin{array}{l}
a=\frac{1-e^{-\kappa^{\mathbb{Q}} \bar{\tau}}}{\kappa^{\mathbb{Q}} \bar{\tau}}, \quad \text { and } \quad \bar{\tau}=30 / 365 \\
b=\left(\theta^{\mathbb{Q}}+\frac{\lambda \mu_{V}}{\kappa^{\mathbb{Q}}}\right)(1-a)+\lambda c \\
c=2\left[\bar{\mu}^{\mathbb{Q}}-\left(\mu_{S}^{\mathbb{Q}}+\rho_{J} \mu_{V}\right)\right]
\end{array}\right.
$$

as shown in Lin (2007) and Duan \& Yeh (2010). It should be stressed here that the VIX squared in Equation (5) is a linear function of the instantaneous variance, $V_{t}$. One can thus take advantage of this linear relationship to calculate the instantaneous variance, $V_{t}$, of the S\&P500, once the VIX value is given.

Carr \& Wu (2006) showed that, under the assumption of no-arbitrage and continuous marking to market, the price of a VIX future, denoted by $F(t, T)$, is a martingale under the risk-neutral probability measure $\mathbb{Q}$. Lin (2007) and Zhang \& Zhu (2006) also concluded that the price of a VIX future is a martingale. Hence the value of a VIX future contract, $F(t, T)$, at time $t$ with settlement at time $T$ is

$$
F(t, T)=E^{Q}\left[\operatorname{VIX}_{T} \mid \mathscr{F}_{t}\right]=E^{Q}\left[\sqrt{a V_{T}+b} \mid \mathscr{F}_{t}\right] \times 100
$$

The expectation in Equation (7) can be explicitly carried out and a closed-form formula for the exact price of a VIX future contract can be obtained, as given by the following proposition: 
Proposition 1 If the S\&P500 index follows the general dynamics given by Equation (4), the conditional probability density function of $V_{T}$, denoted by $p^{\mathbb{Q}}\left(V I X_{T} \mid V I X_{t}\right)$, is given by

$$
p^{\mathbb{Q}}\left(V I X_{T} \mid V I X_{t}\right)=\frac{2 V I X_{T}}{a \pi} \int_{0}^{\infty} \operatorname{Re}\left[e^{-i \phi\left(\frac{V I X_{T}^{2}-b}{a}\right)} f\left(i \phi ; t, \tau,\left(V I X_{t}^{2}-b\right) / a\right)\right] d \phi,
$$

where $\tau=T-t$. The price of a VIX future at time $t$ with maturity $T$ is then:

$$
F\left(t, T, V I X_{t}\right)=\frac{1}{2 \sqrt{\pi}} \int_{0}^{\infty} \frac{1-e^{-s b} f\left(-s a ; t, \tau, \frac{V I X_{t}^{2}-b}{a}\right)}{\sqrt{s}^{3}} d s,
$$

where $f\left(\phi ; t, \tau, V_{t}\right)$ is the moment generating function of the stochastic variable $V_{T}$, given by,

$$
f\left(\phi ; t, \tau, V_{t}\right)=e^{C(\phi, \tau)+D(\phi, \tau) V_{t}+A(\phi, \tau)},
$$

with

$$
\left\{\begin{array}{l}
A(\phi, \tau)=\frac{2 \mu_{V} \lambda}{2 \mu_{V} \kappa^{\mathbb{Q}}-\sigma_{V}^{2}} \ln \left(1+\frac{\phi\left(\sigma_{V}^{2}-2 \mu_{V} \kappa^{\mathbb{Q}}\right)}{2 \kappa^{\mathbb{Q}}\left(1-\mu_{V} \phi\right)}\left(e^{-\kappa^{\mathbb{Q}} \tau}-1\right)\right) \\
C(\phi, \tau)=\frac{-2 \kappa \theta}{\sigma_{V}^{2}} \ln \left(1+\frac{\sigma_{V}^{2} \phi}{2 \kappa^{\mathbb{Q}}}\left(e^{-\kappa^{\mathbb{Q}} \tau}-1\right)\right) \\
D(\phi, \tau)=\frac{2 \kappa^{\mathbb{Q}} \phi}{\sigma_{V}^{2} \phi+\left(2 \kappa^{\mathbb{Q}}-\sigma_{V}^{2} \phi\right) e^{\kappa^{\mathbb{Q}} \tau}} .
\end{array}\right.
$$

The proof of this proposition is left in Appendix A.

Clearly, what has been presented in the proposition is a pricing formula written in the form of an explicit one-to-one function between the VIX futures price and the VIX itself. This formula has several distinctive features. Firstly, it is developed in a general framework that covers most of the known models as special cases, including (i) the Heston (1993) stochastic volatility (SV) model, (ii) the stochastic volatility with jumps in the underlying asset (SVJ) model, (iii) the stochastic volatility model with jumps in the variance process, the SVVJ model, and (iv) the stochastic volatility model with random jumps in both the underlying asset and variance process, the SVJJ model. As a result, the dimensionality of the parameter space is quite high with $\left\{\kappa, \theta, \sigma_{V}, \eta_{V}, \lambda, \mu_{S}, \sigma_{S}, \mu_{V}, \rho_{J}, \eta_{V}\right\}$ as the parameter vector for the most general case $^{2}$. A closed-form and exact pricing formula for VIX based on such a general framework has so far not been seen in the literature.

Secondly, the pricing formula (9) for VIX futures involves a one-dimensional integral with

\footnotetext{
${ }^{2}$ The instantaneous variance, $V_{t}$, is an independent variable, which can be easily calculated from a given VIX value through Equation (5).
} 
its integrand being a well-defined and smooth real function, since it has completely avoided numerically performing the complex-valued Fourier inversion. On the other hand, Zhu \& Zhang (2007) left their final VIX futures pricing formula in the form of two-dimensional integral without evaluating the complex-valued Fourier inversion. Although the parameters in their discussions were assumed to be time-varying in the framework of the Heston SV model, we find that the approach presented in this paper can also be applied to simplify their final solution and avoid the complex-valued Fourier inverse transform. The main disadvantage of a solution being left in terms of complex-valued integrals is that the numerical calculation of these integrals has to be handled carefully as the integrands are multi-valued complex functions. This may cause problems when one has to decide which root is the correct one to take. There have been examples reported in the literature (e.g., Kahl \& Jackel 2005) showing some wrong results when the Fourier inversion is performed numerically. In comparison with those complicated integral calculations, the numerical advantage of our compact solution (9) is obvious. Such an advantage has also been clearly demonstrated by Zhu \& Lian (2010) when they presented an analytical pricing formula for variance swaps.

Thirdly, when the time-to-maturity increases, the prices of VIX futures calculated from the formula (9) become less sensitive to the spot VIX value and fail to capture the evolution of the VIX as the time-to-maturity increases. In the limiting case, the futures prices approach value a constant that is independent of the VIX value, i.e.

$$
\lim _{(T-t) \rightarrow \infty} F(t, T)=\text { Constant }
$$

As will be shown in the empirical studies later, this term structure of VIX futures prices is indeed consistent with the observed traded prices in the CBOE. This feature is quite unique, in contrast to futures contracts written on commodities or equities; the latter always move in a one-to-one fashion with the underlying spot price, even with very a time to expiration.

\subsection{Examination of the Convexity Approximations}

With the newly developed exact pricing formula for VIX futures, it would be of interest to make a comparative study between this formula and two popularly used approximation formulae developed in the past. 
Under the general dynamics as specified in Equation (3), Lin (2007) employed the so-called convexity correction approximation (Brockhaus \& Long 2000). This is essentially the secondorder Taylor expansion of the square root function for the square root of a latent affine stochastic variance process. It is used to calculate the expectation in Equation (7), and obtain an approximation formula for VIX futures in the form:

$$
F(t, T)=E^{\mathbb{Q}}\left[\mathrm{VIX}_{T} \mid \mathscr{F}_{t}\right] \approx \sqrt{E_{t}^{\mathbb{Q}}\left(\mathrm{VIX}_{T}^{2}\right)}-\frac{\operatorname{var}^{\mathbb{Q}}\left(\mathrm{VIX}_{T}^{2}\right)}{8\left[E^{\mathbb{Q}}\left(\mathrm{VIX}_{T}^{2}\right)\right]^{\frac{3}{2}}},
$$

where $\operatorname{var}^{\mathbb{Q}}\left(\mathrm{VIX}_{T}^{2}\right) /\left\{8\left[E^{\mathbb{Q}}\left(\mathrm{VIX}_{T}^{2}\right)\right]^{\frac{3}{2}}\right\}$ is the convexity adjustment relevant to the VIX futures. Naturally, one may wonder if better accuracy can be achieved by extending the second-order convexity correction approximation to a third order in the Taylor expansion of the square root function. Zhang et al. (2010) explored such an extension by carrying out the Taylor expansion of the square root function to the third order and obtained an approximate formula for VIX futures, based on the Heston stochastic volatility model. We now present some numerical comparisons with the results obtained from our exact solution Equation (9) and those from the convexity correction approximations. These comparisons will help readers understand the improvements in accuracy of our exact solution. More importantly, the comparative study presented in this section serves to demonstrate that the convexity approximation technique should be used with care, as it may lead to large errors in some cases.

Figure 1 displays a comparison of the results obtained from our exact formula in the special case of SV model, the exact formula presented by Zhang \& Zhu (2006), the approximation formula presented by Lin (2007) and the approximation formula presented by Zhang et al. (2010), respectively. To produce Figure 1, we chose the parameters to be the same as those presented in the working paper of Brenner et al. (2007), which was an old version of Zhang et al. (2010), i.e. $\kappa=5.5805, \theta=0.03259, \sigma_{V}=0.5885$, and $\sqrt{V_{0}}=8.7 \%$. Since the expression of Zhang et al. (2010) approximation is based on the Heston stochastic volatility model, without jump diffusions, all the parameters relevant to jumps $\left(\lambda, \mu_{S}, \sigma_{S}, \mu_{V}, \rho_{J}\right)$ are set to zero in our numerical examples.

As can be seen in Figure 1, results of our exact formula match with those from Zhang \& Zhu (2006)'s exact formula for VIX futures, demonstrating the correctness of our exact formula. The figure also shows that Lin (2007)'s approximation formula always undervalues VIX futures and 


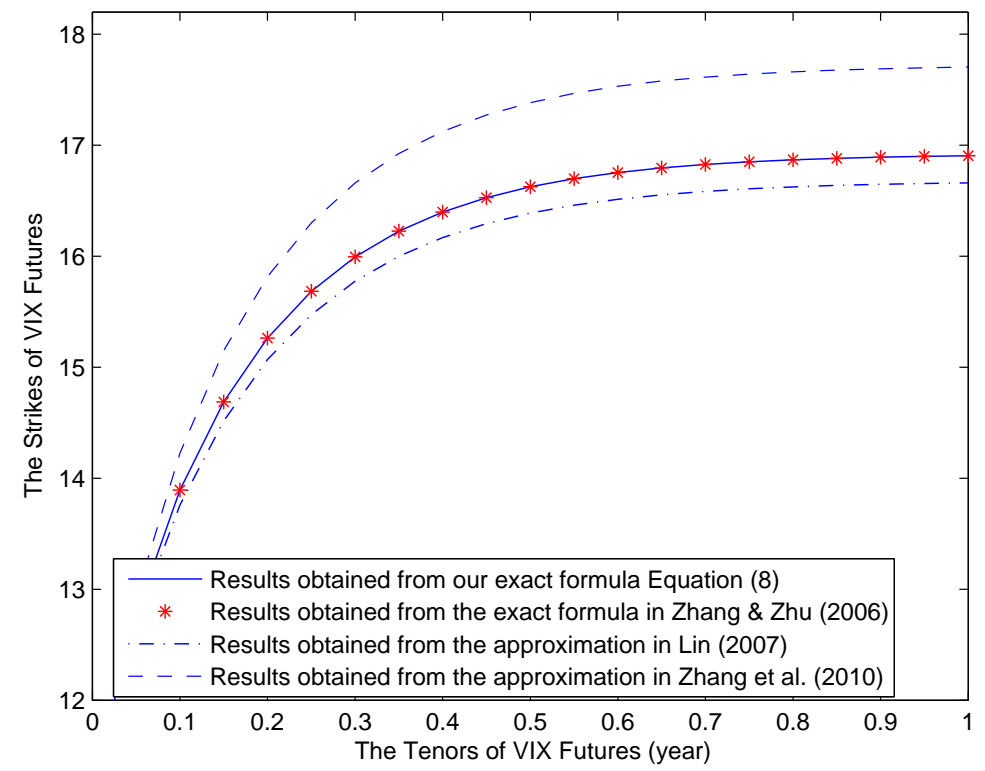

Figure 1: A Comparison of VIX Futures Strikes Obtained from Our Exact Formula and the Two Approximations in the Literature

performs poorly with non-trivial relative pricing errors. For a one-year VIX future, our exact solution produces a value of 16.90 while the second-order convexity approximation results in a value of 16.66 , exhibiting a relative difference of $-1.8 \%$, which is quite large and unacceptable for market traders. In the literature of pricing variance swaps, even when the error level reaches more than $0.5 \%$, Little \& Pant (2001) already declared that it is "fairly large" so that adopting an approximate model to price variance swaps might not be justified. One can also see from this figure that Zhang et al. (2010)'s third-order approximate formula has not only reversed the under-pricing characteristics of the second-order approximation formula but also resulted in some even worse over-pricing errors than Lin (2007)'s second-order approximation formula. Clearly, taking a higher-order approximation does not necessarily lead to a better result when there is a stochastic variable involved.

Of course, it is quite possible that under some other sets of parameters, the two approximations may work well and the relative difference could be substantially smaller than what has been displayed in this particular example. For instance, we have also tested the accuracy of the third-order approximation with the set of parameters presented in Zhang et al. (2010) (i.e., $\kappa=2.4203, \theta=0.03774$ and $\left.\sigma_{V}=0.1425\right)$. Indeed, the relative difference is quite small with these parameters. To explicitly work out the range of validity of the two approximations in the parameter space is a difficult task because of the high dimensionality associated with these ap- 
proximations. Our experience is that the accuracy of these two approximations is sensitive to the parameter $\sigma_{V}$, or the so-called volatility of volatility. The convexity correction approximations work well when $\sigma_{V}$ is sufficiently small. However, when $\sigma_{V}$ has passed a certain threshold (it is roughly 0.5 in this particular example), the deviation resulted from the convexity correction approximation, no matter if it is from the second-order or the third-order approximation, will become unacceptably large. The fact that the accuracies of the both Lin (2007)'s and Zhang et al. (2010)'s approximation formulae are sensitive to the volatility of volatility, $\sigma_{V}$, suggests that adopting the convexity correction approximation based on a Taylor series expansion of square root function is not suitable; this further reinforces the case that exact solutions need to be derived as we have presented in this paper.

\section{Empirical Studies}

Like other pricing formulae, to apply our newly-developed general formula to price VIX futures in practice, one needs to know what parameters to use. The determination of the model parameters in an appropriate way can itself be a difficult problem. The most commonly adopted approaches are i) by minimizing the "pricing errors", measured under some appropriately designed norms, between the model-predicted values and the set of market data; ii) by maximizing "hedging performance" in the sense that the chosen model can render satisfactory hedging performances against specified risks; iii) by producing fit of the model-implied parameters, which are determined from the derivative prices obtained from the model and market data in an "implied" sense, and those determined directly from analyzing the time series of the underlying asset such as the S\&P500 for the case of pricing VIX options or futures. However, implementing any of these approaches usually means that one faces a computationally intensive task, as any routine required to carry out the computational task usually involves millions, if not billions, of iterations. Now, with our closed-form pricing formula which covers four different models, the computation involved in parameter determination will be substantially reduced, thus allowing us to compare which model is the most suitable to price VIX futures. In this section, we present such an empirical study conducted by using the Markov chain Monte Carlo (MCMC) method to estimate the model parameters from the coupled market data of S\&P500 and VIX values and to test the pricing performance of the four models. 


\subsection{The Econometric Methodology}

There are three main reasons why we chose the MCMC method in this study. Firstly, in order to estimate the model parameters, we initially chose the optimization calibration method to infer the parameters by minimizing the squared differences between theoretical values calculated from any VIX futures model and those observed in the market. Our experience is that a minor disturbance in the initial value for the optimization always results in large changes in the solution obtained from the optimization algorithm. In other words, the optimization calibration approach appears to be unstable. The instability may result from the highly nonlinear object function itself, as reported by Zhang \& Lim (2006). Secondly, it has been documented in the literature that the MCMC method has sampling properties superior to other methods. For example, Jacquier et al. (1994) found that the MCMC method outperforms some other statistical inference methods, for example, the generalized method of moments and the quasi-maximum likelihood estimation method, in estimating parameter of stochastic volatility models. Andersen et al. (1999) found that the MCMC method also outperforms the efficient method of moments. Some other advantages (such as computational efficiency, accounting for estimation risk and providing estimations of the latent volatility as well as jumps parameters) are also reported (Eraker et al. 2003). Finally, as noted by Broadie et al. (2007), an efficient estimation procedure should utilize not only the information stored in the underlying asset that varies as a function of time over the period of study, but also the cross-sectional information stored in the derivatives prices over the same period of time. This is a view shared by others (e.g., Pan 2002; Jones 2003; Eraker 2004). In this study, three sets of market data (S\&P500, VIX values and VIX futures prices) were available; simultaneously utilizing these sets of data allows the estimated parameters from the MCMC method to reflect the unbiased information contained in each individual set.

In our study, the MCMC method is implemented by using the software package WinBUGS, which provides an easy and efficient implementation of the Gibbs sampler, and has been successfully applied for a variety of statistic models such as random effects, generalized linear, proportional hazards, latent variables, and even state space models (Yu \& Meyer 2006). Several papers have been appeared which estimate stochastic volatility models using WinBUGS (for example, Meyer \& Yu 2000; Yu 2005; Yu \& Meyer 2006). We have also listed some useful 
websites in Appendix B to provide more information about WinBUGS.

In order to use the MCMC method to estimate the structural parameters and the latent stochastic volatility in our VIX futures pricing model, we construct a time-discretization of Equation (3).

$$
\left\{\begin{array}{l}
Y_{t}=\mu+\sqrt{V_{t-1}} \varepsilon_{t}^{S}+Z_{t}^{S} d q \\
V_{t}=V_{t-1}+\kappa\left(\theta-V_{t-1}\right)+\sigma_{V} \sqrt{V_{t-1}} \varepsilon_{t}^{V}+Z_{t}^{V} d q \\
\operatorname{VIX}_{t}^{2}=\left(a V_{t}+b\right) \times 100^{2}+\varepsilon_{t}^{V I X},
\end{array}\right.
$$

where $\varepsilon_{t}^{S}$ and $\varepsilon_{t}^{V}$ are standard normal random variables with correlation $\rho, Y_{t}$ are continuous daily returns, e.g., $Y_{t}=\ln \left(S_{t} / S_{t-1}\right)$, and $d q=1$ indicates a jump arrival,

One may note that there should be a variance risk premium in the return drift, $\mu+\beta V_{t-1}$. The term $\beta V_{t-1}$ has been ignored in our analysis as the resulting bias is insignificant in daily-interval discretization, consistent with the similar conclusions drawn by Andersen et al. (2002), Pan (2002) and Eraker et al. (2003). Also, it should be noted an additional term which represents the difference between the model-predicted value and the recorded market value, or the so-called "pricing errors", $\varepsilon_{t}^{V I X}$, is introduced in Equation (14). Eraker (2004) adopted a serial dependent $\mathrm{AR}(1)$ model for pricing errors, which is equivalent to assuming that each of the pricing errors follows an independent Ornstein-Uhlenbeck process, based on the prior belief that if an asset is mispriced at time $t$, it is also likely to be mispriced at time $t+1$. In our study, we follow Johannes \& Polson (2002), assuming that $\varepsilon_{t}^{V I X}$ at different $t$ is independent and normally distributed with the zero mean and a known variance, $\sigma_{U}^{2}$. To implement the MCMC inference model, we have also adopted the prior distributions suggested by Eraker et al. (2003) and Eraker (2004) for the unknown parameters.

\subsection{Data Description}

The daily VIX index value and VIX futures prices can be obtained directly from the CBOE. The VIX index data, including the daily open, high, low and close, are available from the January 2, 1990 to the present. And the VIX futures prices, including open, high, low and close and settle prices, as well as the trading volume together with the open interest, are downloadable from the CBOE from March 26, 2004 to the present. In our studies, we use the S\&P500, and VIX daily close levels and VIX futures daily settle prices over the period from March 26, 2004 to July 11, 2008. 


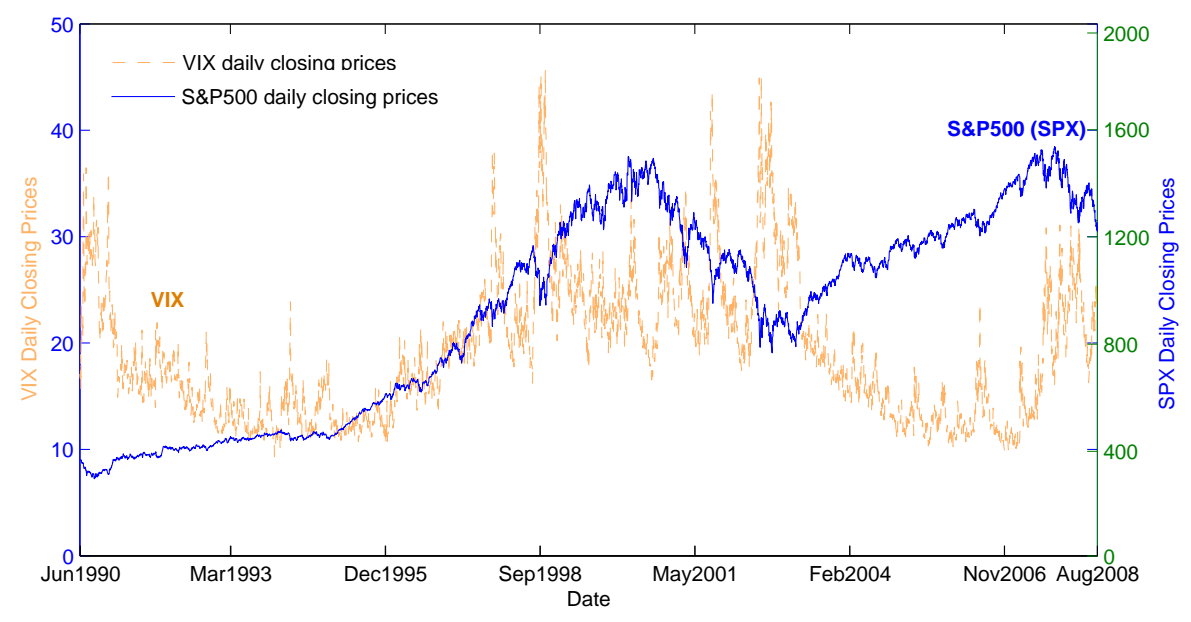

Figure 2: The Historical Data of VIX Index and S\&P500 Index from Jun. 1990 to Aug. 2008

Several exclusion filters were applied to the raw data to construct the VIX futures prices data which are eventually used in our analysis. Firstly, VIX futures that are less than 5 days to maturity were removed from the raw sample to avoid any liquidity-related bias. This is because there are cases in the last few days before expiration when the VIX futures prices move in the opposite direction to the movement of the underlying VIX. This filter principle was also used by Bakshi et al. (1997) and Zhang \& Lim (2006). Secondly, VIX futures data with the associated open interest less than 200 contracts were excluded from the sample to avoid any liquidityrelated bias. Finally, futures prices that are less than 0.5 were not used to mitigate the impact of prices discreteness because of the tick size of 0.01 . This is because most option pricing models assume continuous price movements, whereas in the real world the price moves in ticks. Nandi (1996), Bakshi et al. (1997) and Zhang \& Lim (2006) used this filter rule. In our studies, the minimum futures price in the raw data is 9.95 . So no sample data has been filtered out by this rule. Based on this criterion, we have 6433 VIX futures prices. Because the VIX futures price is independent of the risk-free interest rate, we need not use any interest-rate proxy, such as the LIBOR rate.

Prior to March 26, 2007, the underlying value of VIX futures contract is VIX times 10 under the symbol "VXB", i.e., VXB=VIX $\times 10$. The VIX futures contract size is $\$ 100$ times VXB. For example, with a VIX value of 17.33 on March 26, 2004, the VXB would be 173.3 and the contract size would be $\$ 17,330$. In order to bring the traded futures contract prices in line with the underlying VIX index, CBOE Futures Exchange (CFE) rescaled the VIX futures contracts 
from March 26, 2007, by using the VIX index level as the underlying asset instead of the VXB. At the same time, CFE increased the previous multiplier for the VIX futures contracts from $\$ 100$ to $\$ 1,000$. As a result, the traded futures prices were reduced by a factor of ten and the minimum tick was reduced from $\$ 0.10$ to $\$ 0.01$, but the dollar value of both remained the same. Thus the rescaling did not change the dollar value of VIX futures contracts. The settlement date is usually the Wednesday prior to the third Friday of the expiration month. In our studies, we rescale the VIX futures price in the period from March 26, 2004 to March 25, 2007 by dividing the contract prices by 10 , as guided by the CFE rescaling method ${ }^{3}$.

To illustrate, Figure 2 plots the time series of S\&P500 and VIX index. As can be immediately observed from the figure, the VIX index has a mean-reverting behavior and has a high volatile behavior.

Table 1 provides some basic statistical properties of the S\&P500, VIX index and VIX futures. The futures data are divided into three categories according to the term to expiration as (i) short-term (< 60 days); (ii) medium-term (60-180 days); and (iii) long-term ( $>180$ days). This classification was also used by Lin (2007) in pricing VIX futures and Bakshi et al. (1997) in analyzing S\&P500 options.

Table 1: Descriptive Statistics of VIX and Daily Settlement Prices of the VIX Futures across Maturities

\begin{tabular}{lcccccc}
\hline & & \multicolumn{5}{c}{ Daily VIX futures settlement price } \\
\cline { 5 - 7 } & S\&P500 daily return & VIX value & All & $<60$ days & $60-180$ days & $>180$ days \\
\hline Obs. Number & 1081 & 1081 & 6433 & 2479 & 1868 & 2086 \\
Mean & 0.000268 & 15.63 & 17.89 & 17.03 & 18.53 & 18.34 \\
Median & 0.000432 & 14.02 & 16.31 & 15.13 & 16.50 & 16.73 \\
Std & 0.01012 & 4.74 & 4.16 & 4.56 & 4.18 & 3.39 \\
Minimum & -0.07113 & 9.89 & 10.37 & 10.37 & 12.53 & 13.52 \\
Maximum & 0.05574 & 32.24 & 30.61 & 30.61 & 27.24 & 26.26 \\
Skewness & -0.11414 & 1.22 & 0.60 & 0.82 & 0.44 & 0.77 \\
Kurtosis & 6.61850 & 3.63 & 2.08 & 2.37 & 1.64 & 2.09 \\
\hline
\end{tabular}

Note: The values relevant to S\&P500 return in the above table are quoted using a daily time interval.

\footnotetext{
${ }^{3}$ http://cfe.cboe.com/Data/HistoricalData.aS\&P500
} 


\subsection{Empirical Results}

By implementing the above MCMC procedure in the software package WinBUGS, we obtained the volatility and jump parameters, using the joint data of VIX value and S\&P500 as inputs to estimate the parameters $\Phi$. The estimation procedure was applied to the four models, respectively. Table 2 reports the means and standard deviations of each of the estimated parameters in the four models. All the estimated parameters reported here are annualized.

These reported parameters are quite informative. Table 2 shows that the $\theta$ values are 0.0444 , 0.0424, 0.0409 and 0.0388, respectively, for the SV, SVJ, SVVJ and SVJJ models, which correspond to the annualized long-term volatilities of $21.1 \%, 20.6 \%, 20.2 \%, 19.7 \%$. These estimations are a little higher than the unconditionally sampled standard deviation of S\&P500 return data, which corresponds an annualized value of $16.1 \%$ (see Table 1$)^{4}$. These discrepancies indicate that the sample period for our VIX futures (2004-2008) may be a relatively higher volatile period than that of the S\&P500 (1990-2008). Our estimates for $\theta$ are a little smaller than those reported in Lin (2007), Eraker (2004), Zhang \& Zhu (2006). However, they are very close to the implied estimation in Bakshi et al. (1997). Theoretically, the effective long-term mean variance is $\theta$ for the SV and SVJ models, and $\theta+\frac{\lambda \mu_{V}}{\kappa}$ for SVVJ and SVJJ models. For the SVVJ and SVJJ models, the estimated values of $\theta$ are smaller than those in SV and SVJ models, suggesting that the jump components in volatility processes have indeed captured a portion of the unconditional return variance. This observation is indeed in line with those reported in the literature (e.g., Pan 2002; Lin 2007).

Our estimates of the volatility of volatility $\sigma_{V}$ are a little larger than those obtained by Eraker et al. (2003) from time-series analysis on long-time S\&P500 return, while smaller than those estimated by Eraker (2004) using joint data of return and option prices. These estimates in our study are a little smaller than those in the literature on VIX futures studies, such as Zhang \& Zhu (2006) and Lin (2007). As pointed out by Eraker (2004), there is a disagreement whether estimates obtained previously are reasonable.

Our estimates of the "leverage effect", $\rho$, in the four models range from -0.577 to -0.766 . The absolute values of these estimates are a little larger than those documented in the literature, for example, $\rho=-0.39$ in Jacquier et al. (2004), -0.40 in Eraker et al. (2003), and -0.58 for SVJJ in Eraker (2004). Interestingly, Bakshi et al. (1997) obtained estimates of -0.64, -0.76 and -0.70

\footnotetext{
${ }^{4} 16.1 \%=0.01012 \sqrt{252}$
} 
Table 2: The Parameters of the SV, SVJ, SVCJ, and SVSCJ Models Estimated from the MCMC Method

\begin{tabular}{|c|c|c|c|c|}
\hline Parameters & SV & SVJ & SVVJ & SVJJ \\
\hline$\theta$ & $\begin{array}{c}0.0444 \\
(0.0071)\end{array}$ & $\begin{array}{c}0.0424 \\
(0.0076)\end{array}$ & $\begin{array}{c}0.0409 \\
(0.0071)\end{array}$ & $\begin{array}{c}0.0388 \\
(0.0052)\end{array}$ \\
\hline$\kappa$ & $\begin{array}{c}2.2680 \\
(0.2520)\end{array}$ & $\begin{array}{c}2.2680 \\
(0.2500)\end{array}$ & $\begin{array}{c}1.7640 \\
(0.2520)\end{array}$ & $\begin{array}{c}2.0160 \\
(0.2500)\end{array}$ \\
\hline$\sigma_{V}$ & $\begin{array}{c}0.3856 \\
(0.0504)\end{array}$ & $\begin{array}{c}0.3024 \\
(0.1386)\end{array}$ & $\begin{array}{c}0.3427 \\
(0.1386)\end{array}$ & $\begin{array}{c}0.1134 \\
(0.0252)\end{array}$ \\
\hline$\eta_{V}$ & $\begin{array}{c}-2.0160 \\
(0.5040)\end{array}$ & $\begin{array}{c}-2.5210 \\
(0.5040)\end{array}$ & $\begin{array}{c}-1.7640 \\
(0.2520)\end{array}$ & $\begin{array}{r}-1.7640 \\
(0.2520)\end{array}$ \\
\hline$\rho$ & $\begin{array}{c}-0.7533 \\
(0.0231)\end{array}$ & $\begin{array}{l}-0.6680 \\
(0.0340)\end{array}$ & $\begin{array}{l}-0.7660 \\
(0.0380)\end{array}$ & $\begin{array}{c}-0.5770 \\
(0.0810)\end{array}$ \\
\hline$\lambda$ & & $\begin{array}{c}0.5040 \\
(0.0024)\end{array}$ & $\begin{array}{c}0.2520 \\
(0.0024)\end{array}$ & $\begin{array}{c}0.1764 \\
(0.0010)\end{array}$ \\
\hline$\mu_{S}^{\mathbb{Q}}$ & & $\begin{array}{c}-0.0051 \\
(0.0006)\end{array}$ & & $\begin{array}{c}-0.0074 \\
(0.0007)\end{array}$ \\
\hline$\sigma_{S}$ & & $\begin{array}{c}0.0201 \\
(0.0072)\end{array}$ & & $\begin{array}{c}0.0231 \\
(0.0092)\end{array}$ \\
\hline$\mu_{V}$ & & & $\begin{array}{c}0.0515 \\
(0.0257)\end{array}$ & $\begin{array}{c}0.0094 \\
(0.0012)\end{array}$ \\
\hline$\eta_{J}$ & & $\begin{array}{l}-0.0010 \\
(0.0004)\end{array}$ & & $\begin{array}{l}-0.0022 \\
(0.0004)\end{array}$ \\
\hline$\rho_{J}$ & & & & $\begin{array}{c}0.4216 \\
(0.0336)\end{array}$ \\
\hline
\end{tabular}

Note: This table reports the means and standard deviations (within parentheses) of each estimated parameters in the four models, using the joint data of VIX value and S\&P500. All the estimators reported here are annualized.

for $\rho$ in the SV model, using the data of all options, short-term options and at-the-money options respectively. Lin (2007) presented an estimate of -0.6936 for SVJ model. This disagreement indicates the estimate of $\rho$ is still inconclusive. Fortunately, the estimate of $\rho$ is not so important for the purpose of pricing VIX future, because the VIX and VIX futures are independent of this parameter. 
Table 3: The Comparison of Parameters Estimators for the Stochastic Volatility Model

\begin{tabular}{r|ccccc|l}
\hline Parameters & $\theta$ & $\kappa$ & $\sigma_{V}$ & $\rho$ & $\eta_{V}$ & Methods and Data \\
\hline Our Estimation & 0.0444 & 2.2680 & 0.3856 & -0.7533 & -2.0160 & MCMC 2004-2008 \\
Lin (2007) & 0.1204 & 5.7028 & 0.2689 & -0.5428 & -0.3528 & GMM 2004-2006 \\
Zhang et al. (2010) & 0.0377 & 2.4208 & 0.1425 & $\#$ & $\#$ & Calibration 2004-2008 \\
Zhang \& Huang (2010) & 0.0342 & 1.2929 & $\#$ & $\#$ & -19.1184 & Calibration 2004-2007 \\
Duan \& Yeh (2010) & 0.0472 & 0.8309 & $\#$ & -0.6916 & -11.5905 & Log-likelihood 1990-2007 \\
\hline
\end{tabular}

It is worth noting that even for a simple stochastic volatility model, such as the Heston model, estimates reported in some recent papers adopting various approaches are substantially different from each other. Table 3 shows a comparison of the estimates of the model parameters reported in the literature as well as our own. As can be observed, there are nontrivial differences between them for all estimated parameters, particularly the volatility risk premium parameter, $\eta_{V}$. Just as an issue raised in Zhang \& Huang (2010), we also believe that searching for a reliable estimation method to determine the model parameters from market data remains a challenge. While our work presented in this paper has demonstrated another alternative, complicated stochastic models probably will not gain popularity among market practitioners, until a convincing approach can be accepted and agreed upon by a majority of researchers.

\subsection{Comparative Studies of Pricing Performance}

In this section, we discuss the empirical performance of the four models in fitting the historical VIX futures prices. By following the studies in Lin (2007), we employ three measures of "goodness of fit" (the root mean squared error (RMSE), the mean percentage error (MPE) and the mean absolute error (MAE)) to assess the pricing performance for each of the four VIX futures pricing models. For this purpose, we first compute the model-determined future price using parameters reported in Table 2, then subtract it from its observed market counterpart, to obtain the squared pricing error, percentage pricing error, and absolute pricing error. This procedure is repeated for every future contract and each day in the sample to obtain the mean values of the three tests.

The RMSE, MPE and MAE values for the short-term, mid-term and long-term futures contracts are tabulated in Table 4. Firstly, the RMSE and MAE are the lowest (except the shortterm futures contracts) for the SVJJ model, ranking SVJJ model the best. This suggests that the 
specification benefits are indeed generated by introducing simultaneous jumps in the underlying asset and volatility processes. On the other hand, from the panel of MPE values, in contrast to the above conclusion, the SV model in general outperforms the other three models. Secondly, there appears to be no real advantage with jumps being added to the process describing the dynamics of the underlying asset, as the SVJ model outperforms the SV model only marginally, or performs even worse than SV model according to the MPE test. Thirdly, it is shown that the SVVJ model performs well for the short-term and medium-term futures. However, it significantly overprices the long-term futures with the MPE as high as $10.790 \%$. Finally, all three tests show that the four models perform better for short-term futures than for long-term contracts. For example, the MPE is $3.303 \%$ for short-term futures in the SVJJ model, whereas it increases to $8.942 \%$ for long-term contracts, which is more than doubled. This is also true for other performance measures or other models.

Table 4: The Test of Pricing Performance of the Four Models

\begin{tabular}{lclccc}
\hline & & \multicolumn{3}{c}{ Time to Expiration } & \\
\cline { 3 - 6 } Pricing Errors & Models & All Futures & $<=60$ & $60-180$ & $>=180$ \\
\hline RMSE & SV & 2.668 & 1.782 & 2.940 & 3.230 \\
& SVJ & 2.615 & 1.731 & 2.856 & 3.198 \\
& SVVJ & 2.578 & 1.633 & 2.718 & 3.271 \\
& SVJJ & 2.485 & 1.625 & 2.657 & 3.095 \\
MPE(\%) & & & & & \\
& SV & 5.399 & 2.880 & 5.112 & 8.651 \\
& SVJ & 5.624 & 3.174 & 5.340 & 8.790 \\
& SVVJ & 6.184 & 2.556 & 5.855 & 10.790 \\
& SVJJ & 5.774 & 3.303 & 5.514 & 8.942 \\
& & & & & \\
MAE & SV & 2.343 & 1.479 & 2.713 & 3.037 \\
& SVJ & 2.296 & 1.443 & 2.635 & 3.006 \\
& SVVJ & 2.237 & 1.335 & 2.505 & 3.068 \\
& SVJJ & 2.174 & 1.351 & 2.449 & 2.907 \\
\hline
\end{tabular}

Note: For a given model, we compute the price of each VIX future using the previously estimated parameters reported in Table 2, the current day's VIX and the maturity of the VIX future, then subtract it from its observed market counterpart, to obtain the squared pricing error, percentage pricing error, and absolute pricing error. This procedure is repeated for every future and each day in the sample to eventually obtain the mean values of the three tests.

To illustrate the pricing performance of the various models more clearly, we examine the performance of models in fitting the VIX futures term structure. Following the basic idea of VIX futures term structure proposed by Zhang et al. (2010), we sort all the observed futures prices according to expiration and group these futures by every 30 days to expiration, we then 


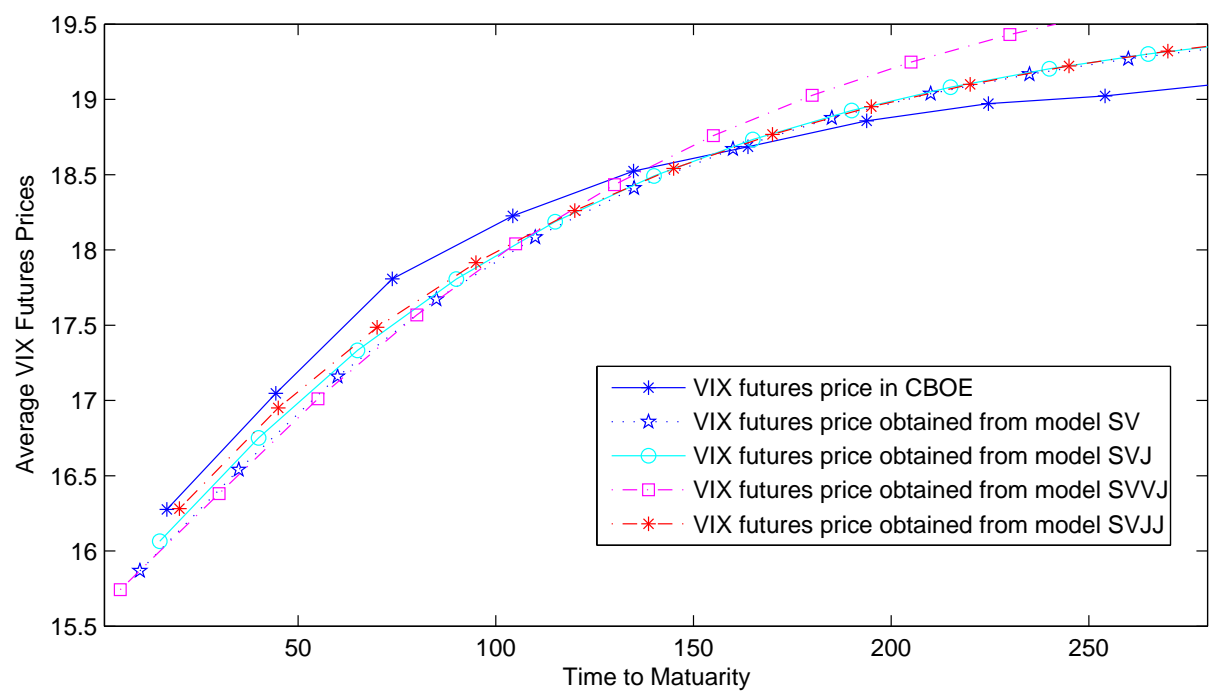

Figure 3: A Comparison of the Term Structures of Average VIX Futures Prices Obtained from Empirical Market Data and the Four Models

compute the average prices of each group. From this procedure, we obtain an empirical term structure of VIX futures, as plotted in Figure 3. Then we compute futures values as a function of expiration, using our empirically obtained parameters in Table 2 with the VIX value in the VIX futures pricing formula (9) being the mean value of VIX 15.63 (see Table 1). Hence we determine four VIX futures term structure curves, corresponding to the four models SV, SVJ, SVVJ, and SVJJ.

It can be observed that the empirical term structure of the VIX futures price, as well as the model-based theoretical term structures is of an upward slope, indicating the short-term mean level of volatility is relatively low compared with the long-term mean level and that the volatility is increasing to the long-term high level. It can be also observed that all term structure curves are concave, and asymptotically approach their upper bounds, indicating that futures prices become less sensitive to time to expiry when the time to expiry increases, and eventually become independent of time to expiry when the time to expiry is large enough. This interesting property, observed from the empirical data of VIX futures, is consistent with our theoretical analysis, Equation (12). As shown in Figure 3, all four models capture the term structure of the VIX futures well. In particular, the SVJJ model performs the best and the SVVJ model the worst; there is a considerable degree of difficulty for the SVVJ model to fit the long-term contracts.

In Figure 4, the model-implied steady-state density distribution for the VIX is compared 
with the empirical frequency of the VIX, which is calculated from the VIX closing levels observed in the CBOE from March 26, 2004 to July 11, 2008. The model implied steady-state density is computed using Equation (8), using the parameters in Table 2. It should be noted that Equation (8) is the conditional transitional probability density, while the empirical VIX frequency is an unconditional one. To obtain the model implied steady-state density distribution of VIX, we let $\tau=T-t$ approach infinity in Equation (8). It can be observed in Figure 4 that none of the four models can capture the "right tail" of the VIX as observed in empirical data. However, the SVJJ model is better than the other three. The SVVJ is again the worst. In the related literature, only Sepp (2008b) and Sepp (2008a) discussed this issue. By calibrating the model to the VIX options data observed on July 25, 2007, Sepp (2008b) obtained his parameters for the same model and derived the VIX density. Unfortunately, his model-implied density cannot capture the right tail feature of the VIX empirical frequency either. Sepp (2008a) estimated the model parameters by minimizing the squared difference between the model and empirical quantiles. In this way, he found the model-implied density fits the empirical counterpart very well. In contrast, we found models based on this set of parameters in Sepp (2008a) cannot capture the VIX futures term-structure as shown in Figure 3. The calculated performance tests (RMSE, MPE and MAE) based on Sepp (2008a)'s parameters are also significantly larger than those in Table 4. It appears difficult to simultaneously fit the VIX futures and VIX values; the implied structural volatility parameters that well fit the derivatives market prices (such as S\&P500 options or VIX futures) cannot describe the corresponding underlying processes (S\&P500, VIX). This is actually a basic question in the empirical literature, as raised by Bates (1996), Bakshi et al. (1997) and Eraker (2004). Although Eraker (2004) found reconciling evidence from spot and option prices by using the MCMC method to infer the related model parameters, we believe that it is still a very difficult problem to obtain parameters which describe simultaneously the VIX and VIX futures.

\section{Conclusion}

In this paper, we have presented a newly-found closed-form, exact solution for VIX futures. The analytic pricing formula has some unique features. Firstly, it is an "umbrella" solution which covers four different stochastic volatility models with or without jumps in both the underly- 


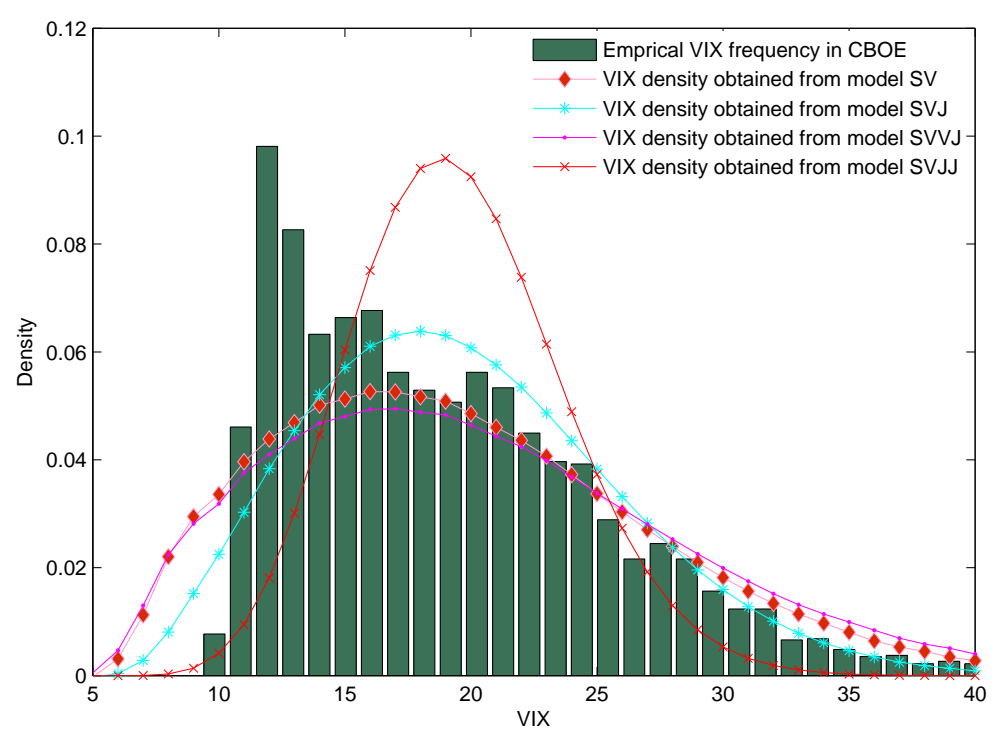

Figure 4: A Comparison of the VIX Steady-State Density Distributions Obtained from the Four Models and Empirical Market Data

ing and volatility processes to describe the S\&P500. Secondly, this formula can be efficiently numerically evaluated as it involves a single integral with a real integrand. With this high computational efficiency, not only is a much shorter computational time needed to compute the price of a VIX futures contract in comparison with the Monte Carlo simulations, the determination of model parameters is also greatly facilitated. Finally, while we have demonstrated that our new formula includes some previously derived formulae (e.g., the SV) as special cases, it has filled a gap as there is no closed-form exact solution available in the literature for some other cases (SVJ, SVVJ and SVJJ). Consequently, we were able to use our formula to examine the accuracy of some analytic approximations previously used for the SVJ, SVVJ and SVJJ cases.

We were also able to use our formula to conduct empirical studies. Using the joint time series data of S\&P500 and VIX, we have demonstrated the determination of model parameters with the MCMC approach through a numerical example. With these parameters extracted from the market data, we then empirically examined the pricing performance of four models (SV, SVJ, SVVJ and SVJJ), taking advantage of our newly-found explicit pricing formula. Our empirical studies show that the Heston stochastic volatility model (SV) describes the dynamics of S\&P500 well and is a good candidate for the pricing of VIX futures. Incorporating jumps into the underlying price can further improve the pricing the VIX futures. However, jumps added to the volatility process appear to add little improvement for pricing VIX futures. 


\section{Appendix A}

Here we give a brief proof of Proposition 1. In order to find a closed-form formula for the exact price of a VIX future contract, we must carry out the expectation in Eq. (7) by explicitly working out the conditional probability density function $p^{\mathbb{Q}}\left(V_{T} \mid V_{t}\right)$. With the instantaneous variance following the stochastic differential equation (SDE) in Eq. (4), the corresponding riskneutral probability density function can be determined by inverting the associated characteristic function.

We consider the moment generating function, $f\left(\phi ; t, \tau, V_{t}\right)$, of the stochastic variable $V_{T}$, conditional on the filtration $\mathscr{F}_{t}$, with time to expiration $\tau=T-t$.

$$
f\left(\phi ; t, \tau, V_{t}\right)=E^{\mathbb{Q}}\left[e^{\phi V_{T}} \mid \mathscr{F}_{t}\right]
$$

Then, the characteristic function is $f\left(\phi i ; t, \tau, V_{t}\right)$. The Feynman-Kac theorem implies that $f\left(\phi ; t, \tau, V_{t}\right)$ is given by thebackward PIDE

$$
\left\{\begin{array}{l}
-f_{\tau}+\kappa^{\mathbb{Q}}\left(\theta^{\mathbb{Q}}-V\right) f_{V}+\frac{1}{2} \sigma^{2} V f_{V V}+\lambda E^{\mathbb{Q}}\left[f\left(V+Z^{V}\right)-f(V) \mid \mathscr{F}_{t}\right]=0 \\
f(\phi ; t+\tau, 0, V)=e^{\phi V}
\end{array}\right.
$$

Following the solution procedure used by Heston (1993), Bakshi et al. (1997), Duffie et al. (2000) and many others, we can solve this PIDE in closed-form by guessing the affine-form solution is,

$$
f\left(\phi ; t, \tau, V_{t}\right)=e^{C(\phi, \tau)+D(\phi, \tau) V_{t}+A(\phi, \tau)} .
$$

By substituting this function into the PIDE, we obtain three ordinary differential equations:

$$
\left\{\begin{array}{l}
D_{\tau}=-\kappa^{\mathbb{Q}} D+\frac{1}{2} \sigma_{V}^{2} D^{2} \\
C_{\tau}=\kappa^{\mathbb{Q}} \theta^{\mathbb{Q}} D \\
A_{\tau}=\lambda E^{\mathbb{Q}}\left[e^{D Z_{t}^{V}}-1 \mid \mathscr{F}_{t}\right]
\end{array}\right.
$$

with the initial conditions

$$
C(\phi, 0)=0, \quad D(\phi, 0)=\phi, \quad A(\phi, 0)=0 .
$$


The solutions to these ODEs are

$$
\left\{\begin{array}{l}
A(\phi, \tau)=\frac{2 \mu_{V} \lambda}{2 \mu_{V} \kappa^{\mathbb{Q}}-\sigma_{V}^{2}} \ln \left(1+\frac{\phi\left(\sigma_{V}^{2}-2 \mu_{V} \kappa^{\mathbb{Q}}\right)}{2 \kappa^{\mathbb{Q}}\left(1-\mu_{V} \phi\right)}\left(e^{-\kappa^{\mathbb{Q} \tau}}-1\right)\right) \\
C(\phi, \tau)=\frac{-2 \kappa \theta}{\sigma_{V}^{2}} \ln \left(1+\frac{\sigma_{V}^{2} \phi}{2 \kappa^{\mathbb{Q}}}\left(e^{-\kappa^{\mathbb{Q}} \tau}-1\right)\right) \\
D(\phi, \tau)=\frac{2 \kappa^{\mathbb{Q}} \phi}{\sigma_{V}^{2} \phi+\left(2 \kappa^{\mathbb{Q}}-\sigma_{V}^{2} \phi\right) e^{\mathbb{Q}_{\tau}}} .
\end{array}\right.
$$

Starting from time $t$, the Fourier inversion of the characteristic function $f\left(\phi i ; t, \tau, V_{t}\right)$ provides the required conditional density function $p^{\mathbb{Q}}\left(V_{T} \mid V_{t}\right)$

$$
p^{\mathbb{Q}}\left(V_{T} \mid V_{t}\right)=\frac{1}{\pi} \int_{0}^{\infty} \operatorname{Re}\left[e^{-i \phi V_{T}} f\left(i \phi ; t, \tau, V_{t}\right)\right] d \phi
$$

The price of a VIX future contract at time $t$ is thus expressed

$$
F(t, T)=E^{\mathbb{Q}}\left[\mathrm{VIX}_{T} \mid \mathscr{F}_{t}\right]=\int_{0}^{\infty} p^{\mathbb{Q}}\left(V_{T} \mid V_{t}\right) \sqrt{a V_{T}+b} d V_{T} \times 100
$$

Schürger (2002) has shown that, after interchanging the expectation and integral using Fubini's theorem, the expectation of the square root function can be expressed as,

$$
E[\sqrt{x}]=\frac{1}{2 \sqrt{\pi}} \int_{0}^{\infty} \frac{1-E\left[e^{-s x}\right]}{s^{\frac{3}{2}}} d s .
$$

Using this identity, Formula (A8) can be simplified as:

$$
F\left(t, T, \operatorname{VIX}_{t}\right)=\frac{1}{2 \sqrt{\pi}} \int_{0}^{\infty} \frac{1-e^{-s b} f\left(-s a ; t, \tau, \frac{V I X_{t}^{2}-b}{a}\right)}{s^{\frac{3}{2}}} d s
$$

Here $f\left(\phi ; t, \tau, V_{t}\right)$ is the moment generating function shown in Eq. (10). This is our pricing formula for VIX futures in the general SVJJ model. A similar technique has also been adopted by Broadie \& Jain (2008) to derive a closed-form pricing formula for volatility swaps.

\section{Appendix B}

BUGS (Bayesian inference using Gibbs sampling) is a user-friendly and freely available software package for the implementation of Bayesian analysis of complex statistical models using Markov chain Monte Carlo (MCMC) methods. BUGS automates the calculation of the full conditional posterior distributions using a model representation by directed acyclic graphs. 
It contains an expert system for choosing an effective sampling method for each full conditional. The software can be downloaded from http://www.mrc-bsu.cam.ac.uk/bugs/welcome.shtml.

The pdf version user manual of the WinBUGS can be downloaded from: http://www.mrcbsu.cam.ac.uk/bugs/winbugs/manual14.pdf

Meyer \& Yu (2000) illustrated the BUGS implementation for a stochastic volatility model using a time series of daily Pound/Dollar exchange rates. The paper is available at http://papers.ssrn.com/sol3/papers.cfm?abstract id=267491.

There are also books on WinBUGS. For example, the book entitled $<<$ Bayesian Modeling Using WinBUGS $>>$ provides an easily accessible introduction to the use of WinBUGS programming techniques in a variety of Bayesian modeling settings, which is available at http://www.amazon.com/gp/search?index=books\&linkCode=qs\&keywords=047014114X.

\section{References}

Andersen, T., Benzoni, L. \& Lund, J. (2002), An empirical investigation for continuous-time equity return models, Journal of Finance 57, 1239-1284.

Andersen, T., Chung, H. \& Sørensen, B. (1999), Efficient method of moments estimation of a stochastic volatility model: A Monte Carlo study, Journal of Econometrics 91, 61-87.

Bakshi, G., Cao, C. \& Chen, Z. (1997), Empirical performance of alternative option pricing models, The Journal of Finance 52, 2003-2049.

Bates, D. (1996), Jumps and stochastic volatility: exchange rate processes implicit in Deutsche market options, Review of Financial Studies 1, 69-107.

Brenner, M., Shu, J. \& Zhang, J. (2007), The market for volatility trading; VIX futures, working paper, available at http://archive.nyu.edu/handle/2451/26289.

Broadie, M., Chernov, M. \& Johannes, M. (2007), Model secification and risk premia: evidence from futures options, The Journal of Finance 62, 1453-1490.

Broadie, M. \& Jain, A. (2008), The effect of jumps and discrete sampling on volatility and variance swaps, International Journal of Theoretical and Applied Finance 11, 761-797. 
Brockhaus, O. \& Long, D. (2000), Volatility swaps made simple, Risk 19, 92-95.

Carr, P. \& Lee, R. (2009), Volatility derivatives, Annual Review of Financial Economics 1, 319339.

Carr, P. \& Wu, L. (2006), A tale of two indices, Journal of Derivatives 13, 13-29.

Cont, R. \& Kokholm, T. (2010), A consistent pricing model for index options and volatility derivatives, Mathematical Finance, (forthcoming) .

Duan, J. \& Yeh, C. (2007), Jump and volatility risk premiums implied by VIX, Journal of Economic Dynamics and Control 34, 2232-2244.

Duffie, D., Pan, J. \& Singleton, K. (2000), Transform analysis and asset pricing for affine jumpdiffusions, Econometrica 68, 1343-1376.

Eraker, B. (2004), Do stock prices and volatility jump? Reconciling evidence from spot and option prices, The Journal of Finance 59, 1367-1404.

Eraker, B., Johannes, M. \& Polson, N. (2003), The impact of jumps in volatility and returns, The Journal of Finance 58, 1269-1300.

Grunbichler, A. \& Longstaff, F. (1996), Valuing futures and options on volatility, Journal of Banking and Finance 20, 985-1001.

Heston, S. (1993), A closed-form solution for options with stochastic volatility with applications to bond and currency options, Review of Financial Studies 6, 327-343.

Jacquier, E., Polson, N. \& Rossi, P. (1994), Bayesian analysis of stochastic volatility models, Journal of Business and Economic Statistics 12, 69-87.

Jacquier, E., Polson, N. \& Rossi, P. (2004), Bayesian analysis of stochastic volatility models with fat-tails and correlated errors, Journal of Econometrics 122, 185-212.

Johannes, M. \& Polson, N. (2002), MCMC methods for continuous-time financial econometrics, Handbook of Financial Econometrics (Elsevier, New York).

Jones, C. (2003), The dynamics of stochastic volatility: evidence from underlying and options markets, Journal of Econometrics 116, 181-224. 
Kahl, C. \& Jackel, P. (2005), Not-so-complex logarithms in the Heston model, Wilmott Magazine pp. 94-103.

Lin, Y. (2007), Pricing VIX futures: Evidence from integrated physical and risk-neutral probability measures, Journal of Futures Markets 27, 1175.

Lin, Y. \& Chang, C. (2009), VIX option pricing, Journal of Futures Markets 29, 523-543.

Lin, Y. \& Chang, C. (2010), Consistent modeling of S\&P 500 and VIX derivatives, Journal of Economic Dynamics and Control 11, 2302-2319.

Little, T. \& Pant, V. (2001), A finite-difference method for the valuation of variance swaps, Journal of Computational Finance 5, 81-101.

Lu, Z. \& Zhu, Y. (2009), Volatility components: The term structure dynamics of VIX futures, Journal of Futures Markets 30, 230-256.

Luo, X. \& Zhang, J. (2010), The term structure of VIX, Working Paper .

Meyer, R. \& Yu, J. (2000), BUGS for a Bayesian analysis of stochastic volatility models, The Econometrics Journal 3, 198-215.

Pan, J. (2002), The jump-risk premia implicit in options: evidence from an integrated timeseries study, Journal of Financial Economics 63, 3-50.

Psychoyios, D., Dotsis, G. \& Markellos, R. (2007), A jump diffusion model for VIX volatility options and Futures, Working paper.

Schürger, K. (2002), Laplace transforms and suprema of stochastic processes, Advances in Finance and Stochastics: Essays in Honour of Dieter Sondermann.

Sepp, A. (2008a), pricing options on realized variance in the heston model with jumps in returns and volatility, Journal of Computational Finance 11, 33-70.

Sepp, A. (2008b), Option pricing: Vix option pricing in a jump-diffusion model, Risk-Londonrisk magazine limited $\mathbf{2 1}, 84$. 
Wang, Z. \& Daigler, R. (2010), The performance of VIX option pricing models: empirical evidence beyond simulation, Journal of Futures Markets, (forthcoming, articles online in advance of print).

Yu, J. (2005), On leverage in a stochastic volatility model, Journal of Econometrics 127, 165178.

Yu, J. \& Meyer, R. (2006), Multivariate stochastic volatility models: Bayesian estimation and model comparison, Econometric Reviews 25, 361-384.

Zhang, J. E. \& Huang, Y. (2010), The CBOE S\&P 500 three-month variance futures, Journal of Futures Markets, 30, 48-70.

Zhang, J., Shu, J. \& Brenner, M. (2010), The new market for volatility trading, Journal of Futures Markets, (forthcoming, articles online in advance of print).

Zhang, J. \& Zhu, Y. (2006), VIX Futures, Journal of Futures Markets 26, 521-531.

Zhang, Z. \& Lim, K. (2006), An Non-lattice pricing model of American options under stochastic volatility, Journal of Futures Markets 26, 417-448.

Zhu, S. \& Lian, G. (2010), A closed-form exact solution for pricing variance swaps with stochastic volatility, Mathematical Finance, (forthcoming, articles online in advance of print).

Zhu, Y. \& Zhang, J. (2007), Variance term structure and VIX futures pricing, International Journal of Theoretical and Applied Finance 10, 111-127. 